\title{
Phase II results in rheumatoid arthritis encouraging for Immunex
}

Following a successful phase II trial, Immunex's (Seattle, WA) Enbrel, a recombinant, human, soluble p75 tumor necrosis factor (TNF) receptor fusion with the human IgG Fc region, appears to be leading the race for new treatments for rheumatoid arthritis. If it is approved on schedule, it will also be the first soluble receptor drug on the market.

A recent 180-patient phase II trial $(N$. Eng. J. Med. 337:141-146, 1997) involving difficult-to-treat rheumatoid arthritis, $75 \%$ of patients receiving the highest dose of Enbrel had significant reductions in disease activity, whereas only $14 \%$ on placebo did so. The side effects were mild, the trial did not reach dose-limiting toxicity, and significantly, no antibodies to the fusion protein were present. Results from a phase III trial will probably be announced this November at the American College of Rheumatology meeting.

Soluble TNF receptors such as Enbrel, Lenercept from Hoffmann-La Roche (Basel, Switzerland), and an unnamed drug from Amgen (Thousand Oaks, CA) are thought to act by "soaking up" excess TNF, thereby preventing it from binding to cell surface TNF receptors. This is thought to interrupt the inflammatory cascade that is central to rheumatoid arthritis and other autoimmune conditions. Enbrel, Lenercept, and Amgen's compound are based on different types of soluble TNF receptor. Enbrel is derived from the class p75 receptor; the other two are both p55 molecules, but they dimerize in different ways.

Immunex believes that the $p 75$ domain is a better disease target because it is known to act as a natural antagonist of TNF. Certain poxviruses carry p75 DNA, which is expressed in infected cells. This results in the production of soluble p75 TNF receptors, decoys that may allow the virus to evade the immune response. "If it's good enough for viruses, it's good enough for us," says Michael Widmer, vice president of biological sciences at Immunex and a researcher on the study. He says that some TNF-dependent T cells activities are also thought to be mediated by 75 .

Two monoclonal antibodies that mop up TNF are also in trials for rheumatoid arthritis. Both of them (like Lenercept) failed to previously treat septic shock. The phase II trials of Centocor's (Malvern, PA) cA2, a humanized chimeric monoclonal, showed that the drug works synergistically with a standard rheumatoid arthritis treatment, methotrexate, but that its effectiveness diminishes upon multiple dosing. There is evidence that antibodies to the drug are formed, a likely cause for its limited efficacy. Centocor is nevertheless currently enrolling its phase III study with $\mathrm{cA} 2$.

Celltech's (Slough, UK) fully humanized antibody, CDP571, seems to be faring somewhat better, at least according to the company's commercial director, Stephen Jones. "Ours is more human than theirs," he says. In a placebo-controlled phase II study conducted in the UK in 1995, doses were well tolerated and showed efficacy ( $B r$. J. Rheumatol. 34:334-342, 1995). An unpublished US study conducted by Celltech's former anti-TNF partner, Bayer (Leverkusen, Germany), used a higher dose also with no discernable side effects. Significantly, says Jones, a number of patients in the UK trial received up to four infusions of the drug over an 8-9 month period and "there was still no problem with immunogenicity."

Immunex's Widmer is not surprised at the problems encountered with some monoclonal antibodies. Whereas soluble receptors are naturally present in the body, antibody-TNF complexes and incompletely humanized antibodies are not, he says.
Celgene (Warren, NJ) is developing a number of new TNF downregulating molecules that are related to, but more powerful than, thalidomide. The molecules (CelSIDS) selectively inhibit phosphodiesterase type 4, which controls the AMP-dependent part of the signaling pathway that downregulates TNF. They have been tested in animal models of rheumatoid arthritis, Crohn's disease, and asthma, and one compound will enter the clinic by the end of 1997, according to Davy Stirling, Celgene's director of biology.

Besides controlling the inflammatory cascade at an earlier point in the cycle, Celgene's small molecule drugs are easier to administer than soluble receptors or anti-TNF antibodies and interfere only with TNF overproduction and not normal TNF production-which is necessary to fight infections. "We believe that there are two different pathways for TNF production," says Stirling. This would mean that Celgene could fine-tune TNF's down-regulation, rather than shutting it down completely, he adds.

Many other companies are addressing the inflammatory cascade of rheumatoid arthritis; they include Immune Response (Carlsbad, CA), Anergen (Redwood City, CA), AutoImmune (Lexington, MA), Isis (Carlsbad, CA), Cadus (Tarrytown, NY), and Tularik.(South San Francisco, CA).

Vicki Brower

\section{Genset-Abbott deal heralds pharmacogenomics era}

On July 28, Genset (Paris) and Abbott Laboratories (Abbott Park, IL) announced the first of what may be a series of deals between specialist genomics companies and pharmaceutical companies for analyzing genetic variations leading to different patient responses to therapeutics-so-called pharmacogenomics. But other genomics companies will need to move quickly: Genset's extensive internal knowledge and integrated large-scale sequencing, mapping, oligonucleotide synthesis, and bioinformatics capabilities may place it in a powerful competitive position.

"The power of pharmacogenomics lies in the ability to identify genes involved in a drug-metabolizing pathway together with the disease genes involved," says Daniel Cohen, chief genomics officer at Genset, "But to do this, you need a systematic genome-wide mapping effort that will enable the dissection of complex gene pathways involved in drug responses and disease." The fact that only 50-100 genes have been correlated with disease using positional cloning approaches based on well-characterized populations of related individuals is a testament to this, he adds.

Pascal Brandys, Genset's chairman and chief executive officer, believes the company's blend of knowledge, manpower, and financial muscle makes it uniquely suited to carrying out this type of large-scale analysis: "We are setting the pace," he says. "Few other 